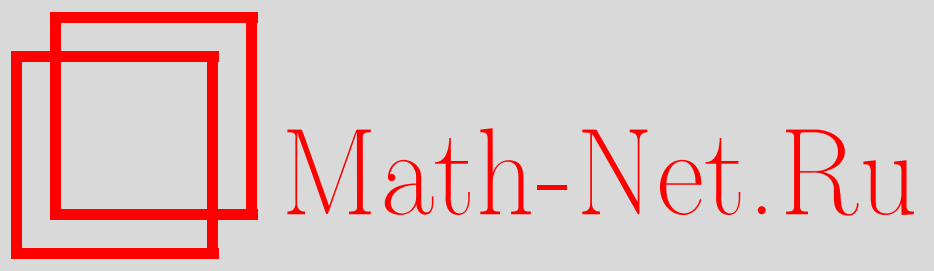

Б. Л. Иоффе, Нарушающие киральность конденсаты в КХД и их связь с решениями кварковых уравнений Дирака с нулевыми собственными значениями, ТМФ, 2012, том 170, номер 2, 165-173

DOI: https://doi.org/10.4213/tmf6756

Использование Общероссийского математического портала Math-Net.Ru подразумевает, что вы прочитали и согласны с пользовательским соглашением http://www.mathnet.ru/rus/agreement

Параметры загрузки:

IP : 44.207 .124 .84

26 апреля 2023 г., 16:18:53

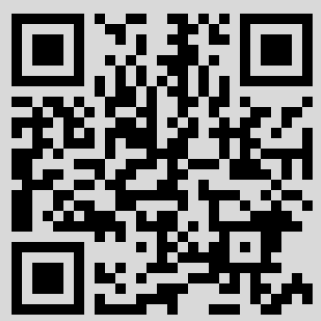




\title{
НАРУШАЮЩИЕ КИРАЛЬНОСТЬ КОНДЕНСАТЫ В КХД И ИХ СВЯЗЬ С РЕШЕНИЯМИ КВАРКОВЫХ УРАВНЕНИЙ ДИРАКА С НУЛЕВЫМИ СОБСТВЕННЫМИ ЗНАЧЕНИЯМИ
}

\begin{abstract}
Показано, что нарушающие киральность конденсаты в безмассовой КХД возникают исключительно из отвечающих нулевому собственному значению решений уравнений Дирака в произвольных глюонных полях. Предложена модель, в которой такие решения являются решениями для кварков, движущихся в инстантонном поле. На основании этой модели для кваркового конденсата вычислены магнитная восприимчивость $\chi$ размерности 3 и магнитные восприимчивости $\kappa$ и $\xi$ размерности 5 . Хорошее соответствие значений $\chi, \kappa$ и $\xi$, полученых с помощью этого подхода, и значений, найденных из адронного спектра, является серьезным аргументом в пользу того, что инстантоны представляют собой единственный источник нарушающих киральность конденсатов в КХД. Обсуждается зависимость кваркового конденсата от температуры. Показано, что фазовый переход, соответствующий температурной зависимости $\alpha(T)$ кваркового конденсата как параметра порядка, представляет собой переход типа кроссовер.
\end{abstract}

Ключевые слова: нулевая мода, инстантон, конденсат.

Посвящается памяти моего друга Альберта Никифоровича Тавхелидзе, выдающегося физика и видного организатора науки в СССР и России

Хорошо известно, что вследствие малости масс легких кварков пертурбативная КХД обладает свойством киральной симметрии, если вкладами тяжелых кварков можно пренебречь, однако в реальном адронном мире киральная симметрия очень сильно нарушена. Это утверждение с очевидностью следует из существования протона с большой массой и отсутствия бариона с отрицательной четностью и массой, равной массе протона. Также хорошо известно, что большие значения конденсатов легких кварков указывают на нарушение киральной симметрии в КХД. Эти два факта имеют глубокую внутреннюю связь: значения массы протона можно выразить через значения кваркового конденсата [1].

* Институт теоретической и экспериментальной физики, Москва, Россия. E-mail: ioffe@itep.ru 
В настоящей работе мы рассматриваем возникновение кваркового конденсата в непертурбативной КХД и устанавливаем связь величины кваркового конденсата с решениями кварковых уравнений Дирака с нулевыми собственными значениями [2]. Таким образом проясняется природа массы протона и, как следствие, природа всех наблюдаемых масс во Вселенной [3].

Рассмотрим действие КХД в евклидовом пространстве-времени:

$$
S=\frac{1}{4} \int d^{4} x G_{\mu \nu}^{2}-\int d^{4} x \sum_{f}\left[\psi_{f}^{+}\left(i \gamma_{\mu} \nabla_{\mu}+i m_{f}\right) \psi_{f}\right]
$$

где $G_{\mu \nu}^{n}$ - тензор глюонного поля, сумма берется по кварковым ароматам,

$$
\nabla_{\mu}=\partial_{\mu}+i g \frac{\lambda^{n}}{2} A_{\mu}^{n}
$$

и $A_{\mu}^{n}$ - глюонное поле. Обратим внимание на то, что в евклидовой формулировке КХД оператор кваркового поля $\bar{\psi}$ заменяется на $\psi^{+}$(евклидову формулировку КХД и инстантонные решения можно найти в работах [4] и [5], см. также обзор [6]). Уравнение Дирака для безмассового кварка в евклидовом пространстве-времени имеет вид

$$
-i \gamma_{\mu} \nabla_{\mu} \psi_{n}(x)=\lambda_{n} \psi_{n}(x)
$$

где $\psi_{n}(x)$ и $\lambda_{n}$ - собственные функции и собственные значения оператора Дирака $-\nabla=-i \gamma_{\mu} \nabla_{\mu}$. Представим операторы кварковых полей как сумму левых и правых операторов:

$$
\psi=\frac{1+\gamma_{5}}{2} \psi_{\mathrm{L}}+\frac{1-\gamma_{5}}{2} \psi_{\mathrm{R}}, \quad \psi^{+}=\frac{1+\gamma_{5}}{2} \psi_{\mathrm{L}}^{+}+\frac{1-\gamma_{5}}{2} \psi_{\mathrm{R}}^{+},
$$

где $\gamma_{5} \psi_{\mathrm{L}}=\psi_{\mathrm{L}}$ и $\gamma_{5} \psi_{\mathrm{R}}=-\psi_{\mathrm{R}}$. Тогда для ненулевых $\lambda_{n}$ лагранжиан и действие сводятся к сумме двух слагаемых:

$$
L=-\int d^{4} x\left[\psi_{\mathrm{L}}^{+} \nabla \psi_{\mathrm{R}}+\psi_{\mathrm{R}}^{+} \nabla \psi_{\mathrm{L}}\right]
$$

Данное выражение полностью симметрично относительно замены $\mathrm{L} \leftrightarrow \mathrm{R}$, поэтому решения уравнений для левых и правых кварковых полей одинаковы - состояния, построенные из левых и правых кварков, полностью симметричны. Это заключение было получено при фиксированном глюонном поле. Очевидно, что усреднение по глюонным полям его не меняет. Совсем другая ситуация возникает в случае $\lambda_{0}=0$. Вклад этого члена в лагранжиан

$$
\Delta L=\int d^{4} x\left[\psi_{\mathrm{L}}^{+}+\psi_{\mathrm{R}}^{+}\right] \nabla \psi_{0}
$$

равен нулю, и нельзя сделать заключения относительно симметрии состояний, построенных из левых и правых кварковых полей. Из сказанного, в частности, следует, что все вакуумные конденсаты в КХД, нарушающие киральность, возникают из решений уравнений Дирака (1), отвечающих нулевому собственному значению. 
Эти общие аргументы подтверждаются хорошо известными фактами. Во-первых, общее представление следа кваркового пропагатора $S(x)$ выражается через спектральную функцию $\rho(\lambda)$, заданную как функцию от собственных значений $\lambda$ (представление Челлена-Лемана):

$$
\operatorname{Tr} S\left(x^{2}\right)=\frac{1}{\pi} \int d \lambda \rho(\lambda) \Delta\left(x^{2}, \lambda\right)
$$

при $x^{2}=0$ функция $\Delta\left(x^{2}, \lambda\right)$ сводится к $\delta(\lambda)$, и мы получаем (в пространстве-времени Минковского) $\rho(0)=-\pi\langle 0|\bar{\psi}(0) \psi(0)| 0\rangle$ (соотношение Банкса-Кашера [7]). Во-вторых, нуль-модовое решение уравнения (1) для безмассового кварка в инстантонном поле представляет собой правую волновую функцию $\psi_{\mathrm{R}}(x)=\left(1-\gamma_{5}\right) \psi(x)$, а в антиинстантонном поле - левую волновую функцию $\psi_{\mathrm{L}}(x)=\left(1+\gamma_{5}\right) \psi(x)$ [8], [9].

Основываясь на этих утверждениях, сформулируем модель для вычисления нарушающих киральность вакуумных конденсатов в КХД. Предположим, что ваку-

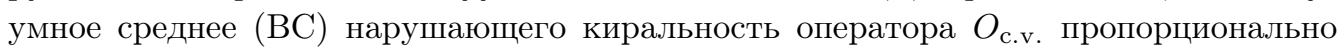
матричному элементу $\psi_{0}^{+} O_{\text {c.v. }} \psi_{0}$, где $\psi_{0}$ - решение уравнения $(1)$ в евклидовом пространстве-времени с нулевым собственным значением, $\lambda_{0}=0$ :

$$
\left\langle 0\left|\bar{\psi} O_{\text {c.v. }} \psi\right| 0\right\rangle \sim \psi_{0}^{+} O_{\text {c.v. }} \psi_{0} .
$$

Функция $\psi_{0}$ зависит от $x$, от положения центра решения $x_{\mathrm{c}}$, а также от размера инстантона $\rho$ : $\psi_{0}=\psi_{0}\left(x-x_{\mathrm{c}}, \rho\right)$. Уравнение (2) следует проинтегрировать по $x_{\mathrm{c}}$, что эквивалентно интегрированию по $x-x_{\text {c }}$ (ниже мы будем использовать обозначение $x$ для $\left.x-x_{\mathrm{c}}\right)$. Предположим, что $\rho=$ const, и найдем его значение из сравнения с известными значениями ВС. Введем в (2) коэффициент пропорциональности $n$. Таким образом, наши предположения приводят к следующему виду ВС:

$$
\left\langle 0\left|\bar{\psi}(0) O_{\text {c.v. }} \psi(0)\right| 0\right\rangle=-n \int d^{4} x \psi_{0}^{+}(x, \rho) O_{\text {c.v. }} \psi_{0}(x, \rho) .
$$

Данная модель аналогична модели разреженного инстантонного газа [10], [11], в которой $x_{\mathrm{c}}$ - положение центра инстантона. В отличие от последней модели, где инстантонная плотность имеет размерность 4 , в нашей модели $n$ имеет размерность 3 и может интерпретироваться как плотность центров нулевых мод в трехмерном пространстве. Заметим, что левая часть равенства (3) записана в пространстве-времени Минковского, тогда как правая часть - в евклидовом пространстве (знак минус введен, чтобы $n$ было положительным). При пертурбативных вычислениях в случае, когда число ароматов $N_{\mathrm{f}}$ больше единицы, вклад инстантонов в действие подавлен множителем, пропорциональным произведению $N_{\mathrm{f}}-1$ масс легких кварков, поэтому выражение (3) означает, что такое подавление отсутствует в непертурбативном вычислении. Зависимость функции $\psi_{0}(x, \rho)$ от $x$ и $\rho$ мы выбрали соответствующей решению с нулевым собственным значением в поле инстантона в цветовой группе $S U(2)$ :

$$
\psi_{0}(x, \rho)=\frac{1-\gamma_{5}}{2} \frac{1}{\pi} \frac{\rho}{\left(x^{2}+\rho^{2}\right)^{3 / 2}} \chi_{0},
$$

где $\chi_{0}$ - спин-цветовая изоспиновая $(|\mathbf{T}|=1 / 2)$ волновая функция, отвечающая равному нулю полному спину $\mathbf{J}=\mathbf{I}+\mathbf{T}$. Функция $\psi_{0}(x, \rho)$ нормирована на единицу:

$$
\int d^{4} x \psi^{+}(x, \rho) \psi(x, \rho)=1 .
$$


Сначала рассмотрим кварковый конденсат $\langle 0|\bar{q} q| 0\rangle$ - наиболее важное нарушающее киральность ВС, определяющее барионные массы [1], [12]-[14]. Здесь $q=u, d$ суть поля $u$-, $d$-кварков. В этом случае $O_{\text {c.v. }}=1$, и в соответствии с $(3),(5)$ мы имеем (на масштабе 1 ГэВ) [5]

$$
n=-\langle 0|\bar{q} q| 0\rangle=(1.65 \pm 0.15) \cdot 10^{-2} \Gamma{ }^{3} \mathrm{~B}^{3},
$$

где интегрирование по подгруппе $S U(2)$ в цветовой группе $S U(3)$, а также антиинстантонный вклад включены в определение $n$. Аномальная размерность кваркового конденсата равна 4/9, и согласно (6) коэффициент $n$ имеет такую же аномальную размерность. Размер $\rho$ волновой функции с нулевым собственным значением можно вычислить в рамках нашей модели ВС. Имеем

$$
-g\left\langle 0\left|\bar{\psi} \sigma_{\mu \nu} \frac{\lambda^{n}}{2} G_{\mu \nu}^{n} \psi\right| 0\right\rangle \equiv m_{0}^{2}\langle 0|\bar{q} q| 0\rangle .
$$

Параметр $m_{0}^{2}$ равен 0.8 ГэВ ${ }^{2}$ на масштабе 1 ГэВ [5], [15]. Аномальная размерность параметра $m_{0}^{2}$ равна $-14 / 27$. Работая в цветовой группе $S U(2)$, заменим $\lambda^{n}$ на $\tau^{a}$, $a=1,2,3$, и возьмем для $G_{\mu \nu}^{a}$ инстантонное поле

$$
G_{\mu \nu}^{a}(x, \rho)=\frac{4}{g} \eta_{a \mu \nu} \frac{\rho^{2}}{\left(x^{2}+\rho^{2}\right)^{2}},
$$

где параметр $\eta_{a \mu \nu}$ был определен в работе [16] (см. также [6]). Подстановка выражений (4) и (8) в уравнение (3) после простых вычислений дает $n / 2 \rho^{2}=m_{0}^{2} n$. Отсюда

$$
\rho=\frac{1}{\sqrt{2} m_{0}}=0.79 \Gamma \ni \mathrm{B}^{-1}=0.256 \text { фм }
$$

(на масштабе 1 ГэВ). Это значение $\rho$ близко к значениям, используемым в моделях разреженного инстантонного газа или инстантонной жидкости. Смешанный кварк-глюонный конденсат (7) был вычислен в работе [17] в модели инстантонной жидкости. Было найдено значение параметра $m_{0}^{2}=1.4 \Gamma_{\ni} \mathrm{B}^{2}$, большее чем значение $m_{0}^{2}=0.8$ Гэ $\mathrm{B}^{2}$, получающееся с помощью правил сумм в КХД.

Теперь вычислим не столь хорошо известные величины - магнитные восприимчивости кваркового конденсата, индуцированные внешним постоянным электромагнитным полем. Магнитная восприимчивость $\chi$ кваркового конденсата размерности 3 определяется формулой [18]

$$
\left\langle 0\left|\bar{q} \sigma_{\mu \nu} q\right| 0\right\rangle_{F}=e_{q} \chi\langle 0|\bar{q} q| 0\rangle F_{\mu \nu}, \quad q=u, d,
$$

где считается, что кварки движутся во внешнем постоянном слабом электромагнитном поле $F_{\mu \nu}$, а $e_{q}$ - заряд кварка $q$ в единицах протонного заряда (протонный заряд $e$ включен в определение $F_{\mu \nu}$ ). Левая часть уравнения (10) нарушает киральность, так что удобно явно выделить множитель $\langle 0|\bar{q} q| 0\rangle$ в правой части. В работе [18] было показано, что $\left\langle 0\left|\bar{q} \sigma_{\mu \nu} q\right| 0\right\rangle_{F}$ пропорционален заряду $e_{q}$ кварка $q$. Универсальная постоянная $\chi$ называется магнитной восприимчивостью кваркового конденсата.

Найдем значение $\chi$ в нашем подходе. Для этой цели необходимо рассмотреть уравнение (1) в присутствии внешнего постоянного электромагнитного поля $F_{\mu \nu}$ 
и найти поправку первого порядка по $F_{\mu \nu}$ к нуль-модовому решению $(4)$. Это нетрудно сделать, представив $\psi$ в виде

$$
\psi(x, \rho)=\psi_{0}(x, \rho)+\psi_{1}(x, \rho)
$$

где $\psi_{0}$ задается уравнением (4), а $\psi_{1}$ представляет собой поправку, пропорциональную $F_{\mu \nu}$. Подставим соотношение $(11)$ в уравнение $(1)$, к которому добавлен член, отвечающий взаимодействию с электромагнитным полем, пренебрежем $\psi_{1}$ в этом члене и решим оставшееся уравнение относительно $\psi_{1}(x, \rho)$. В результате получим

$$
\psi_{1}(x, \rho)=\frac{1}{16} e_{q} \eta_{a \mu \nu} \sigma_{a} F_{\mu \nu} x^{2}\left(1+\frac{1}{2} \frac{x^{2}}{\rho^{2}}\right) \psi_{0}(x, \rho),
$$

где $\sigma_{a}$ - матрицы Паули. Матричный элемент $\psi^{+} \sigma_{\mu \nu} \psi$ оказывается равным

$$
\psi^{+} \sigma_{\mu \nu} \psi=-\frac{1}{2} e_{q} F_{\mu \nu} \psi_{0}^{+} x^{2}\left(1+\frac{1}{2} \frac{x^{2}}{\rho^{2}}\right) \psi_{0}
$$

(мы использовали свойства символов $\left.\eta_{a \mu \nu}[16],[6]\right)$. ВС (10) в пространстве-времени Минковского имеет вид

$$
\left\langle 0\left|\bar{\psi} \sigma_{\mu \nu} \psi\right| 0\right\rangle_{F}=e_{q} F_{\mu \nu} n \frac{1}{\pi^{2}} \int d^{4} x x^{2}\left(1+\frac{1}{2} \frac{x^{2}}{\rho^{2}}\right) \frac{\rho^{2}}{\left(x^{2}+\rho^{2}\right)^{3}}
$$

с учетом условия нормировки $(5)$ для $\psi_{0}(x, \rho)$. Удобно выразить $n$ через кварковый конденсат посредством (6), используя обозначение $x^{2}=r^{2}$, где $r$ - радиус-вектор в четырехмерном пространстве. Тогда согласно (10) имеем

$$
\chi=-\rho^{2} \int_{0}^{R^{2}} d r^{2} r^{4}\left(1+\frac{r^{2}}{2 \rho^{2}}\right) \frac{1}{\left(r^{2}+\rho^{2}\right)^{3}} .
$$

Интеграл (13) квадратично расходится при больших $r$, поэтому мы вводим обрезание $R$. Величину $R$ можно оценить следующим образом. Объем, занимаемый одной нулевой модой в трехмерном пространстве, приближенно равен $1 / n$ (объему ячейки Вигнера-Зейтца). В соответствии с этим квадрат радиуса обрезания в 4-пространстве равен

$$
R^{2}=\frac{4}{3}\left(\frac{3}{4 \pi n}\right)^{2 / 3}=7.92 \Gamma_{\ni} \mathrm{B}^{-2}
$$

где множитель $4 / 3$ соответствует переходу от размерности 3 к размерности 4 . Вычисление интеграла (13) при значениях параметров $\rho$ и $R^{2}$, заданных соответственно в (9) и (14), дает

$$
\chi=-3.52 \Gamma_{\ni} \mathrm{B}^{-2} .
$$

Магнитная восприимчивость кваркового конденсата была ранее вычислена с помощью правил сумм в КХД в работах [19]-[22], где использовались значения масс и констант взаимодействия мезонных резонансов. Недавно получены следующие значения магнитной восприимчивости: $\chi(1$ ГэВ $)=-3.15 \pm 0.3$ Гэ $\mathrm{B}^{-2}[18]$ и $\chi(1$ ГэВ $)=$ 
$-2.85 \pm 0.5 \Gamma_{\ni} \mathrm{B}^{-2}[19]$. Более ранние результаты, полученные с помощью того же метода, таковы: $\chi(0.5$ ГэВ $)=-5.7$ Гэ $\mathrm{B}^{-2}[19]$ и $\chi(1$ ээВ $)=-4.4 \pm 0.4$ Гэ $\mathrm{B}^{-2}[20]$.

Аномальная размерность магнитной восприимчивости $\chi$ равна $-16 / 27$. Она учитывалась в работах [19]-[22], но не в представленном выше вычислении. В некоторых из цитируемых работ также учитывались $\alpha_{s}$-поправка и вклад континуума. Можно считать, что величина (15) относится к масштабу 1 ГэВ, поскольку значение кваркового конденсата (6) относится к этому масштабу, а также поскольку масштаб 1 ГэВ является типичным: на этом масштабе, с одной стороны, нулевые моды и кварковые конденсаты довольно важны (см., например, [14]), с другой стороны, применима модель инстантонного газа [11]. Интеграл квадратично расходится, поэтому оценить точность результата (15) затруднительно. Мы предполагаем, что относительная ошибка не превосходит 30-50\%. В пределах такой ошибки результат (15) согласуется с результатами, полученными с помощью феноменологических подходов. Попытка вычислить магнитную восприимчивость кваркового конденсата размерности 3 была предпринята в работе [23], однако полученные результаты не являются полностью удовлетворительными: они не дают однозначных величин и зависят от масс кварков.

Теперь перейдем к магнитным восприимчивостям $\kappa$ и $\xi$ кваркового конденсата размерности 5, которые были определены в работе [18] формулами

$$
\begin{array}{r}
g\left\langle 0\left|\bar{q} \frac{1}{2} \lambda^{n} G_{\mu \nu}^{n} \bar{q}\right| 0\right\rangle_{F}=e_{q} \kappa F_{\mu \nu}\langle 0|\bar{q} q| 0\rangle, \\
-i g \varepsilon_{\mu \nu \rho \tau}\left\langle 0\left|\bar{q} \gamma_{5} \frac{1}{2} \lambda^{n} G_{\rho \tau}^{n} q\right| 0\right\rangle_{F}=e_{q} \xi F_{\mu \nu}\langle 0|\bar{q} q| 0\rangle .
\end{array}
$$

Сначала вычислим $\kappa$. В этом случае выражение $(12)$ для $\psi_{1}(x, \rho)$ следует умножить на дополнительный множитель $\tau^{b} G_{\mu \nu}^{b} / 2$, где $G_{\mu \nu}^{b}$ задается соотношением (8), а индексы $\mu, \nu$ из (12) заменить на $\lambda, \sigma$. В дальнейших вычислениях мы будем учитывать, что $\chi_{0}$ в (4) соответствует полному спин-цветовому изоспину $J=0$ и, следовательно, $\boldsymbol{\sigma} \boldsymbol{\tau} \chi_{0}=-3 \chi_{0}$ и $\sigma^{a} \tau^{b} \chi_{0}=-\delta^{a b} \chi_{0}$. В соотношении

$$
\eta_{b \mu \nu} \eta_{b \lambda \sigma}=\delta_{\mu \lambda} \delta_{\nu \sigma}-\delta_{\mu \sigma} \delta_{\nu \lambda}+\varepsilon_{\mu \nu \lambda \sigma}
$$

последний член исчезает после суммирования нулевых мод из инстантонной и антиинстантонной конфигураций. Окончательный результат для $\kappa$ имет вид

$$
\begin{aligned}
\kappa & =-\int_{0}^{z} d u \frac{u^{2}}{(u+1)^{4}}\left(1+\frac{u}{2}\right)= \\
& =-\frac{1}{2}\left[\ln (z+1)-\frac{13}{6}+\frac{1}{z+1}+\frac{1}{2(z+1)^{2}}-\frac{1}{3(z+1)^{3}}\right],
\end{aligned}
$$

где $z=R^{2} / \rho^{2}=12.7$. Численные вычисления дают

$$
\kappa=-0.26
$$


Вычисление $\xi$ аналогично вычислению $\kappa$, и в результате получаем

$$
\xi=2 \kappa=-0.52 \text {. }
$$

Значения $\kappa$ и $\xi$ зависят от обрезания лишь логарифмически. Однако, к сожалению, логарифм в выражении (17) не очень велик, и его главная часть компенсирована слагаемым -13/6 в правой части (17), поэтому ошибку выражений (18) и (19) можно оценить приблизительно в 30\%. Магнитные восприимчивости кваркового конденсата размерности 5 были получены с помощью феноменологического подхода из работы [24], основанной на тех же идеях, что и работы [19], [20]. Аномальные размерности не были учтены. В работе [24] был получен следующий результат: $\kappa=-0.34 \pm 0.1, \xi=-0.74 \pm 0.2$. Как можно видеть, он хорошо согласуется с соотношениями (18), (19). Магнитные восприимчивости кваркового конденсата размерности 5 играют важную роль в определении магнитного момента $\Lambda$-гиперона [25].

До настоящего времени рассматривалась модель с одним безмассовым кварком. Если имеется несколько безмассовых кварков $q^{\prime}$ (как в реальном мире), то в инстантонной плотности появляется дополнительный множитель, стремящийся к нулю при $m_{q^{\prime}} \rightarrow 0$. Появление этого множителя установлено в теории возмущений, но можно ожидать, что он будет отсутствовать в непертурбативных подходах. Так, в работе [26] показано, что при учете кваркового конденсата вместо массы кварка $m_{q^{\prime}}$ в плотности инстантона появляется множитель $m_{q^{\prime}}-\left(2 \pi^{2} / 3\right)\langle 0|\bar{q} q| 0\rangle \rho^{2}$, отличный от нуля при $m_{q^{\prime}} \rightarrow 0$.

Подведем итог. Показано, что как следствие решения уравнения Дирака с нулевым собственным значением для безмассового кварка в произвольном глюонном поле возникает нарушение киральной симметрии в КХД. Предложена модель, аналогичная модели разреженного инстантонного газа, в котором решение с нулевым собственным значением то же, что и в поле инстантона. Параметры модели - плотность нулевых мод и их размер - определяются из значений кваркового и кварк-глюонного конденсатов. Вычисленные в рамках данной модели значения магнитных восприимчивостей кварковых конденсатов размерностей 3 и 5 согласуются с результатами, полученными методом правил сумм в КХД с использованием свойств адронного спектра. Согласие этих двух подходов представляет весомый аргумент в пользу того, что инстантоны являются единственным источником нарушающих киральность конденсатов в КХД.

Обратимся теперь к зависимости кваркового конденсата, рассматриваемого как параметр порядка, от температуры. Ожидается, что кварковый конденсат обращается в нуль при высоких температурах, а киральная симметрия восстанавливается. Обычно обсуждаются две возможности: фазовый переход второго порядка и фазовый переход типа кроссовер. В рамках нашего подхода условие нормировки (5) имеет место при любой температуре [27], поэтому зависимость кваркового конденсата от температуры сводится к $\alpha(T) \equiv\langle 0|\bar{q} q|\rangle_{T}=n(T)$. Можно ожидать, что $n(T)$ обращается в нуль, только если квантовое число, которое несет инстантон, обращается в нуль. Это квантовое число представляет собой топологический заряд, который не зависит от температуры, поэтому при нашем подходе $n(T)$ никогда не обращается в нуль, и фазовый переход является переходом типа кроссовер. Зависимость параметра порядка от температуры для фазового перехода второго порядка 
вблизи критической точки $T_{\text {с }}$ сглаживается флуктуациями [28] (аналогичное утверждение относится, конечно, и к кроссоверу), определяющимися длинноволновыми колебаниями полей. В КХД они задаются низкочастотным глюонным полем $G_{\mu \nu}^{n}$, которое является постоянным. Имеем

$$
\frac{\Delta \alpha\left(T_{\mathrm{c}}\right)}{\alpha\left(T_{\mathrm{c}}\right)} \sim\left\langle 0\left|G_{\mu \nu}^{2}\right| 0\right\rangle T_{\mathrm{c}}^{2} \rho^{6},
$$

где $\Delta \alpha\left(T_{\mathrm{c}}\right)$ имеет порядок величины вариации $\alpha(T)$ вблизи критической точки кроссовера. Очевидно, что левая часть соотношения (20) должна обращаться в нуль, если $T_{\mathrm{c}}=0$. Мы полагаем, что множитель, соответствующий данному требованию, есть $T_{\mathrm{c}}^{2}$. Множитель $\rho^{6}$ добавлен в $(20)$ из соображений размерности.

Рассмотрение зависимости $\alpha(T)$ целиком основано на неявном допущении, что $\alpha$ имеет физический смысл при конечных $T$. Однако неясно, как можно определить $\alpha(T)$ при конечных $T$. Мне не удалось сформулировать мысленный эксперимент, в котором $\alpha(T)$ можно было бы измерить при конечных $T$, поэтому, возможно, такой объект не имеет физического смысла при $T \neq 0$.

Благодарности. Работа выполнена при финансовой поддержке РФФИ (грант № 09-02-00732) и частичной поддержке CRDF Cooperative Program (грант RUP22961-MO-09). Автор благодарит за поддержку European Community-Research Infrastructure Integrating Activity "Study of Strongly Interacting Matter" в paмкаx VII Framework Program of EU.

\section{Список литературы}

[1] B. L. Ioffe, Nucl. Phys. B, 188:2 (1981), 317-341; Erratum, 191:2 (1981), 591-592.

[2] B. L. Ioffe, Phys. Lett. B, 678:5 (2009), 512-515, arXiv: 0906.0283.

[3] Б. Л. Иоффе, УФН, 176:10 (2006), 1103-1104, arXiv: hep-ph/0601250.

[4] M. Shifman (ed.), Instantons in Gauge Theories, World Scientific, River Edge, NJ, 1994.

[5] B. L. Ioffe, V.S. Fadin, L. N. Lipatov, Quantum Chromodynamics: Perturbative and Nonperturbative Aspects, Cambridge Monographs on Particle Physics, Nuclear Physics, and Cosmology, 30, Cambridge Univ. Press, Cambridge, 2010.

[6] А. И. Вайнштейн, В. И. Захаров, В.А. Новиков, М. А. Шифман, УФН, 136:4 (1982), $553-591$.

[7] T. Banks, A. Casher, Nucl. Phys. B, 169:1-2 (1980), 103-125.

[8] L. S. Brown, R. Carlitz, C. Lee, Phys. Rev. D, 16:2 (1977), 417-422.

[9] R. Jackiw, C. Rebbi, Phys. Rev. D, 16:4 (1977), 1052-1060.

[10] D. I. Diakonov, V. Yu. Petrov, Phys. Lett. B, 147:4-5 (1984), 351-356.

[11] T. Schäfer, E. V. Shuryak, Rev. Modern Phys., 70:2 (1998), 323-425, arXiv: hep-ph/9610451.

[12] B. L. Ioffe, Z. Phys. C, 18:1 (1983), 67-68.

[13] B. L. Ioffe, ЯФ, 72:7 (2009), 1263-1270, arXiv: 0810.4234.

[14] B. L. Ioffe, Prog. Part. Nucl. Phys., 56:1 (2006), 232-277, arXiv: hep-ph/0502148.

[15] В. М. Беляев, Б. Л. Иоффе, ЖЭТФ, 83:3 (1982), 876-891.

[16] G. 't Hooft, Phys. Rev. D, 14:12 (1976), 3432-3450.

[17] M. V. Polyakov, C. Weiss, Phys. Lett. B, 387:4 (1996), 841-847, arXiv: hep-ph/9607244.

[18] B. L. Ioffe, A. V. Smilga, Nucl. Phys. B, 232:1 (1984), 109-142.

[19] В. М. Беляев, И. И. Коган, ЯФ, 40 (1984), 1035-1038. 
[20] I. I. Balitsky, A. V. Kolesnichenko, A. V. Yung, ЯФ, 41 (1985), 282-284.

[21] P. Ball, V.M. Braun, N. Kivel, Nucl. Phys. B, 649:1-2 (2003), 263-296, arXiv: hep-ph/0207307.

[22] J. Rohrwild, JHEP, 09 (2007), 073, 17 pp., arXiv: 0708.1405.

[23] H.-C. Kim, M. Mussakhanov, M. Siddikov, Phys. Lett. B, 608:1-2 (2005), 95-106, arXiv: hep-ph/0411181.

[24] I. I. Kogan, D. Wyler, Phys. Lett. B, 274:1 (1992), 100-110.

[25] J. Pasupathy, J. P. Singh, S. L. Wilson, C. B. Chiu, Phys. Rev. D, 36:5 (1987), 1442-1450.

[26] M. A. Shifman, A. V. Vainshtein, V. I. Zakharov, Nucl. Phys. B, 163 (1980), 46-56.

[27] D. J. Gross, R. D. Pisarski, L. G. Yaffe, Rev. Modern Phys., 53:1 (1981), 43-80.

[28] Л. Д. Ландау, Е. М. Лифшиц, Теоретическая физика, т. 5: Статистическая физика, Наука, М., 1976. 